Future Studies, Mental Health and the Question of Citizenship

\begin{tabular}{|r|l|}
\hline Journal: & Mental Health and Social Inclusion \\
\hline Manuscript ID & MHSI-11-2019-0038 \\
\hline Manuscript Type: & Opinion Piece \\
\hline Keywords: & Mental Health, Citizenship, Future Studies, Futurology \\
\hline & \\
\end{tabular}

SCHOLARONE $^{\mathrm{m}}$
Manuscripts $^{\text {Manus }}$ 


\title{
Future Studies, Mental Health and the Question of Citizenship.
}

\begin{abstract}
Purpose: This paper seeks to examine the value of utilising future studies to explore citizenship for people with mental health challenges.
\end{abstract}

Design/methodology/approach: This paper critiques the discipline of future studies and considers it in the context of the citizenship and mental health literature. It explores how future studies can be utilised to promote marginalised voices, such as those of people with mental health challenges.

Findings: Technology is leading to rapid change in society including what it means to be a citizen (Isin and Nielsen 2008; Isin and Ruppert 2015). Whilst citizenship has been promoted within mental health for a long time, change has been slow (Rowe and Davidson, 2016). In order to create inclusive opportunities for people with mental health challenges, any focus on citizenship in mental health needs to not only address the present time but to anticipate and influence future technological directions.

Originality/value: This paper is original in bringing together mental health and the future impact on society of new technologies. It stands to offer a new perspective to discussions on citizenship.

Key Words: Mental Health, Citizenship, Future Studies, Futurology

\section{Introduction}

Digital technologies are impacting on every sphere of life, including employment, leisure, transport, relationships, health care and education. All are changing at a rapid pace (Chace 2018; Harari 2016). As digital technologies are changing everyday occurrences, they are influencing what it means to be a citizen. New practices of citizenships are emerging including digital citizenship (regular users of the internet who engages in politics and social movements via information technology), consumer citizens (people who define their citizenship through their purchasing choices), global citizens (people who define their citizenship through a sense of shared humanity and roles and responsibilities that transcend the nation state) (Isin and Nielsen 2008; Isin and Ruppert 2015).

Future Studies includes disciplines such as sociology, media, cultural, technology and business studies, and makes predictions on changes to society based on economics, climate, demographics, political theory, and developments in information technology (Potts 2018). Whilst predominantly used in business, planning and policy making, as an academic study there are opportunities to adopt critical sociological perspectives (Potts, 2018; Bergman et al, 2014). There is also a growing body of popular Future Studies literature that is exploring the likely changes of society due to technological advances and how this will impact on citizens (Chace, 2018; Harari, 2016).

In terms of civil and human rights, people with mental health challenges continue to be significantly excluded from participation in society and experience stigma and discrimination (Slade et al, 2017). This results in social exclusions that perpetuate and sustain inequalities. Recent enquiries into the causes of mental ill health have identified the role of structural inequalities which has led to a renewed call to examine citizenship so that people with mental health challenges are able to access the same opportunities and enjoy the same 
rights as other citizens (Hamer and Finlayson 2015; Hamer 2012; Hamer et al. 2018; Harper et al. 2017; Rowe 2015; Rowe and Davidson 2016; Slade et al. 2017). It could be argued people with mental health challenges have a unique experience of citizenship, as their human rights are dependent their health status, for example when detained under the Mental Health Act (Brannelly, 2018; Hamer and Finlayson 2015; Vervliet et al. 2017). Due to these fundamental concerns, citizenship is long fought for, a contested concept and challenged within the survivor movement. Despite this the pace of change has been slow. Neither the de-institutionalisation in the 1980s and 1990s, nor the Recovery movement have delivered the level of equalities required (Eiroa-Orosa and Rowe, 2017; Rowe and Davidson, 2016). Now, there is another ubiquitous issue that needs to be considered in this debate, and that is the role of technologies.

Due to the impacts of technology society is changing rapidly. This paper will argue that it is important that citizenship for people with mental health challenges is not just explored in the present but also to look at future impacts. Currently, the development of this tech-led future society is driven by industry (Potts, 2018), to the exclusion of other voices (Bergman et al, 2014). People with mental health challenges, as a marginalised population, are excluded from contributing and influencing the conversation about citizenship, rights, the use of technologies and the future. The technology industry focus is on people with higher levels of social connectivity and social capital to monetarise their social value, and these industries are less interested in marginalised populations (Skeggs and Yuill 2016).

Bergman et al, (2014) argue that part of the Futures Studies approach is that there are opportunities for marginalised voices to play an active role in shaping futures, for example, feminist Future Studies approaches challenge existing power structures. It can also be a vehicle for expressing constructive hope when dealing with complex social issues such as climate change (Ojala 2015). This paper explores literature relating to Future Studies, citizenship and mental health and poses the question do Future Studies have a role in promoting citizenship for people with mental health challenges?

\section{Visions of the Future}

One consensus in the Future Studies literature is that over the next 20-40 years (2020 2040) there will be significant advances in technologies which will influence what it means to be a citizen (Chace, 2018; Harari, 2017; Potts, 2018). Typically, these tend to be dystopian or utopian predictions. Dystopian futures are characterised by increased social control and inequality where the privileged elite have access to the benefits of a technologically driven society or even a society where Artificial Intelligence (Al) itself takes control of society and dictates to humans (see for example Harari, 2016). Utopian futures are described as heralding a new age of democracy, equality and creativity due to technology (Bregman, 2017).

Calum Chace (2018), in his book Surviving Al, describes the following future scenario where a character called Julia describes a day in her life, summarised below:

Julia wakes up refreshed having been supported by her digital assistant to monitor her sleep patterns. Her health is monitored and ensures she is healthy and well. Based on her morning health readings, the digital assistant provides advice on diet and what to eat for breakfast. Overnight a drone has delivered a new outfit for work...Whilst she commutes, her personal assistant helps her prepare for work and suggests news stories of interest. As the journey continues she relaxes by wearing a virtual reality (VR) headset allowing her to look at the journey as if it had been in the Victorian era....At work, she uses the latest psychological evaluation algorithm to understand how best to communicate with the people in her virtual meeting. Using 
VR technology she communicates with people across the world. After dark, on the way home from work she has her personal assistant checking the street CCTV cameras to ascertain the safest way home.

Whilst this is a fictional account, it paints a picture of how technologies may impact on employment, housing, health, transport, leisure and security. Perkins and Morgan (2017) argue that if people with mental health challenges, in line with the social model of disability, had greater access to social supports or adjustments this could empower people to access the opportunities open to all in society. If technologies were harnessed to promote inclusivity they could provide some of this additional support. Many of these new technologies are already with us or are under development. For example, digital innovations within mental health care include chatbot therapy, smart phones being used to monitor relapse from psychosis, wearable technologies to monitor physical and emotional health, diagnosis through the use of Al predictive technologies, monitoring for suicidality on social media and remote stress or symptom monitoring. People with mental health challenges are already interacting with technology to monitor and support mental wellbeing. For example, people are using Alexa or Google Home to discuss their emotional distress (Luxton et al. 2016; Miner et al. 2017; Poulin et al. 2016; Tal and Torous 2017).

These developments, not just in healthcare but across wider society are raising new ethical questions about the impact on citizens' rights, in particular around surveillance, privacy, data and algorithmic programming (Harari, 2017 ; Luxton et al. 2016; Poulin et al. 2016). This is particularly important for marginalised groups, such as people with mental health challenges, who already struggle to access their full rights as citizens. There is also evidence that people with mental health challenges are disadvantaged by some of the algorithms programmed into social media (Skeggs and Yuill 2016). People with mental health problems are one of a number of groups who are more likely to be digitally excluded (do not have access to or are unable to access the internet). Therefore, they may not have the opportunity to engage in digital citizenship (Greer et al. 2019).

As well as ethical challenges these technologies have the potential to benefit the lives of citizens. For example, work may become less important as Al takes on more traditional employment roles and there may be a greater focus on happiness and wellbeing rather than measuring a nations success on Gross Domestic Product (Bregman, 2017; Harari, 2016). This is something that Slade et al (2017) have advocated in regards to promoting the mental health of the whole population.

\section{Citizenship}

In Western society, citizenship has conventionally been the relationship between the individual and the state and the balance between the rights and responsibilities of each (Ponce and Rowe, 2018.) This definition and balancing of rights and responsibilities are based on social norms and favour some groups over others and can sustain oppression (Atterbury and Rowe 2017; Vervliet et al. 2017). In the U.K. in particular this tradition has grown out of the work of T.H. Marshall and his seminal work from 1949 Citizenship and Social Class (Marshall, 1987) and the establishment of the welfare state. He describes how people have civic, political and social rights. However, the tradition has been criticised from a neoliberal perspective arguing the welfarist approach has been replaced by individuals taking personal responsibility (Atterbury and Rowe, 2017). It has also been criticised by feminists and post-colonialists for its gendered and ethnocentric stance (Hamer et al, 2018). It was never intended to take into account the needs of people with disabilities or mental health problems, it was designed to support the employed when not in employment (Atterbury and Rowe, 2017). 


\section{Citizenship and People with Mental Health Challenges}

Ponce and Rowe (2018) argue that by critiquing citizenship and understanding who is included and excluded, the concept of citizenship can be used as a vehicle for social change by exposing the processes of exclusion and advocating for marginalised groups. There has been a long-standing movement within mental health, and in particular the survivor movement to promote citizenship and equal rights. This can be traced back to the development of moral treatment in the $19^{\text {th }}$ Century, through deinstitutionalisation in the $20^{\text {th }}$, to the current Recovery movement (Rowe and Davidson 2016). Despite this focus each of these approaches has failed to deliver access to full citizenship for people with mental health challenges (Rowe and Davidson, 2016; Slade et al, 2017). This is largely thought to be due to the dominance of the combination of systematic injustices and the dominance of the abnormality approach to mental health. People with mental health challenges disproportionately experience the injustices of unemployment, forced treatment and compulsion, poverty, and hardship (Brannelly 2018). These injustices do not stand alone; they intersect with other forms of discrimination and structural inequalities relating to ageism, disablism, poverty, racism, sexism, homophobia, and transphobia. When mental health is approached primarily from a clinical standpoint these problems become located in the individual rather than people's psychological and social context (Faulkner, 2017; Rowe and Davidson, 2016).

Faulkner (2017) argues this long-standing view of locating the problem within the individual has been reinforced through the dominant ideology of neoliberalism. This, she argues, not only links to clinical understanding of recovery but also to those ideas of personal recovery leading to an over reliance on promoting personal responsibility to the exclusion of understanding the impact of the material conditions and context within which the person lives. This is happening despite the origins of the Recovery approach being within the service-user survivor movement. There are claims these attempts to promote citizenship have been "colonised" by policy makers to harness the values of empowerment and mutual support to cut services and blame individuals for their problems (Eiroa-Orosa and Rowe 2017). Faulkner (2017), Rowe and Davidson, (2016) and Slade et al, (2017) state that in order to address these inequalities it is essential that approaches to citizenship and mental health have the voice and experience of people with mental health challenges at their centre and that solutions need to be led by or co-produced with people with mental health challenges and be linked to the wider disability struggle and identity politics.

Rowe and Davidson (2016) promote a citizenship framework to tackle these injustices. Their citizenship framework is defined by the 5 Rs of rights, responsibilities, roles, resources, and relationships that a democratic society makes available to its members through public and social institutions, social networks, and everyday social interactions. Rowe and Davidson argue that services should promote citizenship as the foundation of recovery from mental health challenges not the end point. They propose a twin focus for mental health services to support this agenda. Firstly, by undertaking community development work and secondly, developing a citizenship education programme for people accessing such services.

They have also developed a measure and training programme which they are in the process of piloting. It evaluates both individual support towards citizenship and community change.

\section{Acts of Citizenship and People with Mental Health Challenges}

Hamer et al (2017) and Brannelly (2018) take a different position on understanding and promoting citizenship within mental health services. Rather than looking at the 5 Rs as a 
citizenship framework they explore Isin's work on Acts of Citizenship (Isin and Nielsen 2008). Isin and Nielsen (2008) and Isin and Ruppert (2015) argue that citizenship should not be constituted as a single definition or even multiple definitions but instead should be understood as Acts of Citizenship. They critique traditional approaches to citizenship linked primarily to the relationship between the individual and the state, as reductionist, arguing people are citizens across multiple domains such as global citizens, consumer citizens, digital citizens. They suggest that it is more useful to explore acts of citizenship as a concept in its own right outside of the usual definitions of citizenship. Acts of Citizenship are described as the events through which people constitute themselves as citizens and this creates a pluralistic understanding. They claim that such acts involve both responsibility and answerability, but are irreducible to either. As Acts of Citizenship are either collective or individual deeds that rupture social-historical patterns, they are able to lead to positive change for marginalised groups. This provides opportunities for feminist and post-colonial critical perspectives. Therefore, they transform people from being passive subjects to political actors creating new ways of being as citizens (Hamer et al, 2017).

Hamer et al (2017) used this framework to interview peer workers and professionals working within inpatient mental health services. They explore how mental health workers promote inclusion by subverting rules and organisational practices as existing practices and structures do not effectively support peoples' access to full citizenship. Hamer et al. (2017) argue that by making these practices visible this can lead to organisational change. They argue a citizenship-based rights framework for mental health workers can embedded and prioritised to support inclusion and citizenship.

Brannelly (2018) through a study titled 'Acts of Citizenship' explored the priorities of service user activists campaigning for change in mental health service provision. The service user activists from New Zealand and England - unequivocally their priorities for change were the removal of the use of force, compulsory treatment and detention. The study questions the ways in which people experience a lack of care within mental health services and question the lack of progress within service provision. The work explores which practices are just or not and how the marginalisation and subjugation of certain groups can be challenged through Acts of citizenship.

Whilst none of the research outlined here explores the impact on citizenship of future technologies, it does outline priorities to support people's participation as full citizens. These are: rights and care in relation to compulsion, and alternatives to restrictive practices, access to relationships, employment, housing and leisure activities, freedom from discrimination and stigma. They also highlight the importance of understanding how these issues intersect across all protected characteristics.

As the pace of social change for people with mental health challenges is slow it could be argued that it is essential to explore both the impact of future technological advances and the changing multi-dimensional nature of citizenship. If there is not a future focus, the developments on citizenship within mental health could be misaligned with the direction of society or miss opportunities to contribute to these discussions. This is especially important as it is not just governments but also large corporations, in particular technology companies, such as Google or Facebook that are shaping the future (Potts, 2018) and in turn include and exclude the voices that contribute to those discussions (Bergman et al, 2014), and who gets access to data for research (Edwards and Brannelly 2017).

\section{Future Studies and Mental Health}


Whilst there are numerous studies looking at future developments in mental health (a search of Medline and CINAHL returns 100,000 articles) the utilisation of the academic approaches of Future Studies have only had limited application within mental health. These studies have focused on population changes in relation to dementia and not changes to society due to technology (Abramson and Halpain 2002; Connolly 2012). Whilst they do not focus on citizenship they do demonstrate the value of future thinking and planning in relation to dementia. They also show the limitations of the approach in the challenge of only being able to see things in the context of your own time and context, as the emphasis is on professional solutions rather than technological or community solutions. Connolly (2012) argues that in mental health Future Studies is under used and even there is a focus on the future it looks at immediate threats and consequences, not the medium to longer term future.

One exception to this, whilst not a research paper, is a Youtube video created by a service user activist O'Hagan (2013), who imagined a radio interview set in 2042 which describes the repeal of the Mental Health Act. This approach has a lot in common with the Future Studies approach of back casting (Davidson, 2017) and gives concrete examples of how current United Nations human rights documents can shape the future. As often with service user led research, rather than the focus being clinical it is on how society and the state responds to people in distress (Faulkner, 2017). Within the video there is very little on the use of technology and how this could impact on restrictive practices within mental health services. However, this is a good example of how future thinking approaches can contribute to discussions on citizenship and human rights in both the present and the future. Within the Future Studies literature itself mental health is very much explored through the lens of clinical recovery and the need to change peoples' brains rather than the need to also change society (Bregman, 2017; Chase, 2018; Harari, 2016).

\section{The Value of Future Studies}

One of the key debates within Future Studies is the accuracy of prediction. Lee (2012) makes the claim that the future is knowable and that future studies should be considered a science. He argues that approaches that look for multiple perspectives are pessimistic and too concerned with uncertainty. (Potts 2018) counters Lee's argument arguing that the evidence is not there to back up his claims and that people only really have the capacity to see things from the perspective of their own time, for example people expecting to have Jet Packs in the $21^{\text {st }}$ Century rather than predicting smart phones. (Odeleye 2015) argues that Lee is overly optimistic about the ability of Future Studies to accurately predict the future and that Lee is too dismissive of post-modern approaches in being able to highlight complexities. They go on to argue that by treating Future Studies as predictive and not exploring the disadvantages Future Studies may bring, a full exploration is not undertaken for the benefits and threats across different sectors and therefore maintains the status quo. If Future Studies is not approached from a critical standpoint there is a danger that it may lead to self-fulfilling prophesies (Odeleye, 2015).

One of the challenges within the Future Studies literature is that it does not always explicitly articulate the political standpoint of the author especially those that take neoliberalism as a neutral standpoint. This maintains current political categories, choices and conflicts present in current power relations and gender dynamics (Bergman et al, 2014). Gunnarsson-Östling et al. (2012) state gender perspectives or perspectives of marginalised groups are rare within Future Studies and are often side tracked. Because of this it has been argued that Futures Studies does not have value for feminists. Bardzell (2018) and Bergman et al. (2014) counter the argument that future studies does not have value for marginalised groups by suggesting that if feminists do not explore future thinking, the possibility of bring about more equal futures is diminished. They also argue that without embracing feminist 
methodologies Future Studies become monolithic and maintain existing power structures and that these feminist methodologies can lead to more critical and reflexive positions within Future Studies research and could radically change the dynamics of the field. Bardzell (2018) explicitly calls for feminist participative action in relation to the development of technologies.

It is important to note, that whilst feminism has not linked strongly with the academic discipline of Future Studies, future thinking has played a strong role in feminism in critiquing and challenging the status quo. Perhaps no more so than in Donna Haraway's seminal book, from 1985, the Cyborg Manifesto (Haraway, 1994). In the Cyborg Manifesto, using blasphemy and irony, she breaks down the ideas of division between humans, machines and animals by exploring inter-species and technological kinship and how this challenges existing power structures and categorisations (for example, between machine/living organism). Latimer (2017) describes how Haraway rages at technoscience, that whilst it has scope for inclusion, it is at the heart of division. Through the metaphor of the cyborg Haraway critiques traditional conceptualisations of gender, sexuality, humanity, nature and technology. Both Sofoulis (2015) and Latimer (2017) argue that revisiting these ideas from the context of the $21^{\text {st }}$ Century provides a new perspective on the work. Sofoulis (2015) highlights the importance of having values-based rather than positivist approaches to science and technology. Latimer (2017) calls for direct challenge of current divisions with more inclusive and collaborative visions for the future.

As well as Future Studies, in regards to mental health, it may be worth learning from other approaches to future thinking from groups who experience marginalisation. Capers (2019) puts forward a similar argument, to that of Bergman et al (2014), for Afrofuturism.

Afrofuturism is a unique discipline and is described as the intersection between black culture, technology, liberation, imagination and mysticism which can bridge the future and the past. As well as an academic discipline it is expressed through literature, art, music and film. It thereby helps reimagine the experience of people of colour providing an opportunity for black communities and African diaspora to engage in potential, real and imagined futures that can rupture the status quo, providing a link between the past, present and future (Womack 2013). It could be argued that similar themes are important when advocating for equality for people with mental health challenges. Both Afrofuturism and Haraway's Cyborg Manifesto show the importance of creativity in imagining futures and breaking down taken for granted power structures.

Bergman et al. (2014) propose that the purpose of utilising future studies with feminist methodologies is not to create a singular unified vision for feminist futures but rather to open new ways of thinking and innovative approaches. They suggest there are multiple ways of exploring feminist futures. These could be: imaging what the future could look like; describing preferred scenarios; working as "alarm clocks" [sic] if the future is not wanted or as a way of showing things are on the right track. Future Studies can encourage students to express constructive hope about controversial social problems such as climate change rather than denying them or feeling passive (Ojala 2015). There are specific teaching techniques within future studies such as discussion, role play and back casting (identifying a potential desirable future and then working backwards to identify the steps required to reach it) (Davidson, 2017). Gunnarsson-Östling et al. (2012) argue that participatory research methods are best suited to developing alternative futures, thus there is a commonality between Future Studies and participatory research methods. Both seek to transition students or research participants from knowledge consumers to knowledge creators (Davidson, 2017). 


\section{Discussion:}

It is clear there is a need for further research into citizenship for people with mental health challenges. There are a range of voices articulating that an increased focus on citizenship is required to challenge and change people's experience within both mental health services and society in general (Eiroa-Orosa and Rowe 2017; Faulkner 2017; Hamer et al. 2018; Rowe and Davidson 2016; Slade et al. 2017). It could be argued that the needs of people with mental health challenges in the present are so acute that exploring the future is a privileged indulgence which will be of limited immediate benefit and that all research should focus on promoting citizenship in the here and now. This focus on citizenship in the here and now should include ethical considerations in relation to technology particularly in relation to surveillance, privacy, and algorithmic programming.

It is not proposed that Future Studies should be the only focus of citizenship research in mental health but rather it may complement existing directions. In both feminist Future Studies and Afrofuturism it is argued exploring the future it is possible to impact positively on the present (Caper, 2019; Bardzell 2018). In addition, it is important to note technologies are not the only impact on citizenship. Rowe and Davidson (2016) describe the importance of paying attention to the material, social, cultural, and political environment in which recovery and citizenship can be impeded or nurtured. On-going austerity, climate crisis and demographic changes are also significant and may be more or equally important issues for people with mental health challenges to explore.

Digital technologies are changing society and what it means to be a citizen across multiple domains and these changes are taking place rapidly. Technological companies and policy makers already utilise Future Studies or future thinking approaches to ensure they anticipate challenges and set the direction for their own developments. If the same approaches are not explored for their validity within mental health this could lead to the rights and needs of people being left even further behind. Whilst there are very few studies in both gender studies and mental health research, there are indications that Future Studies could have value in supporting conversations around future societal change and engage and amplify diverse voices that are currently missing from conversation about the future (Bergman et al, 2014). In particular it is crucial that those who experience the greatest exclusions and rights violations due their mental health status are included, in particular those from the Black and Minority Ethnic community or LGBTQI community and those involved in the wider disability struggle (Slade et al, 2017). With the development of "Mad Studies" (Faulkner, 2017) lessons could be drawn from Afro-futurism to develop "mad-futurism" led by people with mental health challenges. Drawing learning from feminist future thinking participatory methods may be beneficial in developing research in this area. It would also suggest with the critiques of neoliberalism in the development of citizenship for people with mental health challenges feminist and post-colonial approaches would provide useful critical perspectives.

As technologies are impacting on what it means to be a citizen and are being delivered in healthcare it is more important than ever that people who experience mental health challenges are included and have the opportunity to lead these developments. This is not just in the testing of the technology but in the ethical considerations and the setting of agendas within the development of digital technologies within mental health. This means not just seeking engagement and partnership with mental health services and policy makers but also digital technology companies. At present within the literature there has not been a comprehensive review of the level of involvement of people with lived experience in the development of digital mental health products. It is evident one of the key priorities for citizenship for people who experience mental health challenges is around coercion and unforced treatment (O'Hagan 2013; Wilson et al. 2016). Therefore, it may be important to explore ethical technological solutions to promoting citizenship in this area. Also it will be important to include technology (and future technologies) in any proposed citizenship 
frameworks for people experiencing mental health challenges (Hamer et al, 2017; Rowe and Davidson, 2016).

As described above citizenship is a complex and contested concept. In order to discuss the future technological impacts on citizenship it is important to conceptualise citizenship which is flexible to change and provides a framework for challenge for people from marginalised communities. It could be argued Isin's "Acts of Citizenship" has clear advantages over the other approaches to citizenship outlined. Firstly, it has a broader definition of citizenship and incorporates broader forms of citizenship such as digital and consumer citizens. Secondly, it positions the individual as a political actor and defines acts of citizenship as acts that rupture the status quo rather than the individual experiencing citizenship as tension between the state and individual. It could be argued that participating in Future Studies research and contributing to generating new knowledge is an Act of Citizenship in itself.

As well as exploring the notion of citizenship, in order to explore future citizenship it may be worth exploring the following questions:

- What are the likely technological impacts on citizenship?

- Whose voices are being heard in the development of technology?

- What threats and opportunities do technological changes present for people with mental health challenges?

- How do each of these threats and discrimination intersect with other forms of discrimination and oppression?

- What would desirable future citizenship look like?

- What steps would need to be taken to achieve this?

- What would be the "alarms" that it is off track and what can be the responses?

\section{Conclusion}

Does Future Studies have a role in promoting citizenship for people with mental health challenges? This was the question posed in the Introduction. This paper argues that it does, particularly as society is changing quickly and changes for citizenship for people with mental health challenges have been slow. Future studies can provide a framework to prepare for these changes, express constructive hope and be a way of sounding alarms. It is important to acknowledge the limitations of Future Studies; that it is not predictive and people can only see things from the context of their own time, nevertheless it can be useful to explore and critique power dynamics and create opportunities for marginalised communities. It is important not just to look at the future, many of the technologies are already under development or are being applied within mental health care. It is essential to understand what extent are people with mental health challenges are involved in setting the agenda for new innovations and then the extent to which they are co-developed. It is recommended a scoping literature review is undertaken to explore this. It is also important to continue to be aware of the digital exclusion of people with mental health challenges and the reasons for and consequences of this. The other key consideration both now and in the future is the ethical implications of digital technology and big data in relation to all citizens but in particular those from marginalised groups such as people with menta health challenges.

It is vital people with mental health challenges are able to lead and contribute to discussions that shape future citizenship. Participating in these discussions could be considered as an Acts of Citizenship as they have the potential to expose inequality and contribute to discussions on social change. In relation to digital technologies it is also important within this to explore engagement with technology companies and how these conversations can be 
shared and promoted across all stakeholders, including mental health professionals, family members and the wider community.

\section{References}

Abramson, Tobi A. and Maureen Halpain. (2002). "Providers of Mental Health Services-Now and in the Future." Generations 26(1):107-110.

Atterbury, Kendall and Michael Rowe. (2017). "Citizenship, Community Mental Health, and the Common Good." Behavioral Sciences \& The Law 35(4):273-287.

Bardzell, Shaowen. (2018). "Utopias of participation: Feminism, design, and the futures."

ACM Transactions on Computer-Human Interaction 25(1).

Bergman, Helena, Kristina Engwall, Ulrika Gunnarsson-Östling and Livia Johannesson.

(2014). "What about the Future? The Troubled Relationship between Futures and

Feminism." NORA: Nordic Journal of Women's Studies 22(1):63-69.

Brannelly, Tula. (2018). "An ethics of care transformation of mental health service provision: Creating services that people want to use." In Ethics from the ground up: emerging debates, changing practices and new voices in healthcare, ed. J. Wintrup, Biggs, H., Brannelly, T., Fenwick, A., Ingham, R., Woods, D: Palgrave MacMillan.

Bregman, Rutger. (2017). Utopia for Realists. Boston: Little, Brown and Company. Capers, I. Bennett. (2019). "Afrofuturism, Critical Race Theory, and Policing in the Year 2044." New York University Law Review (Issue 1):1.

Chace, Calum. (2018). Surviving Al. 2nd Edition. Great Britain, 3Cs.

Connolly, Moira. (2012). "Futurology and mental health services: Are we ready for the demographic transition?" The Psychiatrist 36(5):161-164.

Edwards, Rosalind and Tula Brannelly. (2017). "Approaches to democratising qualitative research methods." Qualitative Research 17(3):271-277.

Eiroa-Orosa, Francisco José and Michael Rowe. (2017). "Taking the Concept of Citizenship in Mental Health across Countries. Reflections on Transferring Principles and Practice to Different Sociocultural Contexts." Frontiers In Psychology 8:1020-1020.

Faulkner, Alison. (2017). "Survivor research and Mad Studies: the role and value of experiential knowledge in mental health research." 4 Edition. Great Britain: Taylor \& Francis. Greer, Ben, Dan Robotham, Sara Simblett, Hannah Curtis, Helena Griffiths and Til Wykes. (2019). "Digital Exclusion Among Mental Health Service Users: Qualitative Investigation." Journal of Medical Internet Research 21(1):8-8.

Gunnarsson-Östling, Ulrika, Åsa Svenfelt and Mattias Höjer. (2012). "Participatory methods for creating feminist futures." Futures 44:914-922.

Hamer, H. P. and M. Finlayson. (2015). "The rights and responsibilities of citizenship for service users: Some terms and conditions apply." Journal of Psychiatric and Mental Health Nursing 22(9):698-705.

Hamer, Helen Paris. (2012). "Inside the city walls: Mental health service users' journeys towards full citizenship." THESIS available at:

https://researchspace.auckland.ac.nz/bitstream/handle/2292/11915/whole.pdf?sequence=2 (Accessed $1^{\text {st }}$ November 2019)

Hamer, Helen Paris, Michael Rowe and Carol Ann Seymour. (2018). "'the right thing to do': Fostering social inclusion for mental health service users through acts of citizenship." International Journal of Mental Health Nursing.

Harari, Yuval N. (2016). Homo deus : a brief history of tomorrow. London: Vintage. Haraway, Donna. (1994). "A cyborg manifesto: Science, technology, and socialist-feminism in the late twentieth century." In Theorizing feminism: Parallel trends in the humanities and social sciences., eds. Anne C. Herrmann and Abigail J. Stewart. Boulder, CO: Westview Press.

Harper, Annie, Liat Kriegel, Christina Morris, Helen P. Hamer and Matthew Gambino. (2017). "Finding citizenship: What works?" American Journal of Psychiatric Rehabilitation 20(3):200217. 
Isin, Engin F. and Greg Marc Nielsen. (2008). Acts of Citizenship. London: Zed Books. Isin, Engin F. and Evelyn Sharon Ruppert. (2015). Being digital citizens: Lanham : Rowman \& Littlefield Publishers, 2015.

Latimer, Joanna. (2017). "Review: Donna J Haraway, Manifestly Haraway: The Cyborg Manifesto, The Companion Species Manifesto, Companions in Conversation (with Cary Wolfe)." Theory, Culture \& Society 34(7/8):245-252.

Lee, Michael. (2012). Knowing our Future: the Startling Case for Futurology. Oxford: Infinite Ideas.

Luxton, David D., Susan Leigh Anderson and Michael Anderson. (2016). "Ethical issues and artificial intelligence technologies in behavioral and mental health care." In Artificial intelligence in behavioral and mental health care., ed. David D. Luxton. San Diego, CA: Elsevier Academic Press.

Marshall, T. H. (1987). Citizenship and Social Class. London: Pluto Press.

Miner, Adam S., Arnold Milstein and Jefferey T. Hancock. (2017). "Talking to Machines

About Personal Mental Health Problems." JAMA: Journal of the American Medical

Association 318(13):1217.

O'Hagan, Mary. 2013. "Radio interview in 2042 - Mary O'Hagan on the new mental health 'care without coercion' laws." Available at: https://www.youtube.com/watch?v=Tle1trJhs2g (access $1^{\text {st }}$ November, 2019)

Odeleye, Dellé. (2015). "Knowing Our Future: The Startling Case for Futurology."

KronoScope 15(2):253-257.

Ojala, Maria. (2015). "Hope in the Face of Climate Change: Associations With

Environmental Engagement and Student Perceptions of Teachers' Emotion Communication

Style and Future Orientation." Journal of Environmental Education 46(3):133-148.

Perkins, Rachel and Phil Morgan.( 2017). Promoting Recovery throughout our Lives: SAGE. Ponce, Allison N. and Michael Rowe. (2018). "Citizenship and community mental health care." American Journal of Community Psychology 61(1-2):22-31.

Potts, John. (2018). "Futurism, Futurology, Future Shock, Climate Change: Visions of the Future from 1909 to the Present." PORTAL: Journal of Multidisciplinary International Studies 15(1/2):99-116.

Poulin, Chris, Paul Thompson and Craig Bryan. (2016). "Public health surveillance:

Predictive analytics and big data." In Artificial intelligence in behavioral and mental health care., ed. David D. Luxton. San Diego, CA: Elsevier Academic Press.

Rowe, Michael. (2015). Citizenship and mental health. New York, NY: Oxford University Press.

Rowe, Michael and Larry Davidson. (2016). "Recovering Citizenship." The Israel Journal Of Psychiatry And Related Sciences 53(1):14-20.

Skeggs, Beverley and Simon Yuill. (2016). "Capital experimentation with person/a formation: how Facebook's monetization refigures the relationship between property, personhood and protest." 3 Edition. Great Britain: Taylor \& Francis.

Slade, Mike, Lindsay Oades and Aaron Jarden. (2017). Wellbeing, recovery and mental health. New York, NY: Cambridge University Press.

Sofoulis, Zoë. (2015). "The Cyborg, its Manifesto and their relevance today: Some reflections." PLATFORM: Journal of Media \& Communication 6(2):8-15.

Tal, Amir and John Torous. (2017). "The digital mental health revolution: Opportunities and risks." Psychiatric Rehabilitation Journal 40(3):263-265.

Vervliet, Marianne, Didier Reynaert, An Verelst, Sofie Vindevogel and Jessica De Maeyer. (2017). "'If you can't follow, you're out' the perspectives of people with mental health problems on citizenship." Applied Research in Quality of Life.

Wilson, Stacey C., Jenny Carryer and Tula Brannelly. (2016). "New risks: the intended and unintended effects of mental health reform." Nursing Inquiry 23(3):200.

Womack, Ytasha. (2013). Afrofuturism : The World of Black Sci-fi and Fantasy Culture. Chicago: Independent Publishers Group. 
